\title{
The Connection Whose Holonomy is the Classical Adiabatic Angles of Hannay and Berry and Its Generalization to the Non-Integrable Case
}

\author{
R. Montgomery \\ M.S.R.I., 1000 Centennial Dr., Berkeley, CA 94720, USA
}

\begin{abstract}
We show how averaging defines an Ehresmann connection whose holonomy is the classical adiabatic angles which Hannay defined for families of completely integrable systems. The averaging formula we obtain for the connection only requires that the family of Hamiltonians has a continuous symmetry group. This allows us to extend the notion of the Hannay angles to families of non-integrable systems with symmetry. We state three geometric axioms satisfied by the connection. These axioms uniquely determine the connection, thus enabling us to find new formulas for the connection and its curvature. Two examples are given.
\end{abstract}

\section{Contents}

Introduction . . . . . . . . . . . . . . . . . . . . . . . . . . . . 269

1. Families of Hamiltonian Group Actions . . . . . . . . . . . . . . . . . . 271

2. Defining the Connection and Relating it to Hannay. . . . . . . . . . . . . . 273

3. Geometric Properties; Main Results . . . . . . . . . . . . . . . . . . 277

4. Some Examples, Including the Foucault Pendulum . . . . . . . . . . . . . 280

5. Families of Completely Integrable Systems . . . . . . . . . . . . . . . . 283

6. Example: Family of Linear Oscillators . . . . . . . . . . . . . . . . . 287

7. Proofs of Theorems 2 and Proposition 1. . . . . . . . . . . . . . . . . . . . . . 289

8. Generalizations and Future Directions . . . . . . . . . . . . . . . . . 291

References. . . . . . . . . . . . . . . . . . . . . . . 293

\section{Introduction}

In (1984) Berry discovered a phase associated to a family of self-adjoint operators. This phase is determined by a loop in the space which parametrizes the family, and by initial choice of nondegenerate eigenvalue. In (1985) Hannay found a classical analogue of this phase. This analogue, called the classical adiabatic angles, or Hannay angles, is associated to a family of completely integrable systems. The 
angles are determined by a loop in parameter space, together with initial values for the action variables.

Simon (1983) gave a simple geometric framework for Berry's quantum phase. The original goal of our paper was to provide an analogous geometric framework for the classical adiabatic angles. In such a framework the angles (or phase) are the holonomy of a connection for a bundle over parameter space.

Our formula (2.1) defines such a connection, which we call the Hannay-Berry connection. In the words of Cushman, this connection is given by declaring that "averaging defines parallel translation."

From a local point of view, formula (2.1) is not new: Hannay defined his angles by integrating the local equations of parallel translation thus obtained. However, by expressing the connection in invariant language, we were led to the following new results:

(A) The Hannay-Berry connection is defined even if the family of Hamiltonians is not completely integrable. One only needs the family to admit a continuous group of symmetries, the group one averages over.

(B) The connection obeys three geometric axioms (see Sect. 3.1):

(1) parallel translation preserves the functions $I$ which generate the symmetry group (i.e. the components of the associated family of moment maps),

(2) parallel translation is obtained by integrating the flow of a time-dependent Hamiltonian vector field,

(3) a normalization for the corresponding Hamiltonians.

These three axioms uniquely determine the connection: if there is such a connection, then it is the Hannay-Berry connection. The necessary and sufficient condition for the existence of such a connection is the "adiabatic constancy" of the moment map $I$, which we write $\left\langle d_{M} I\right\rangle=0$.

Result (B), together with a new formula for the connection's curvature, is summarized in Theorem 2, Sect. 3.

Alan Weinstein suggested the normalization axiom (3) in the completely integrable case. The reader may also wish to see Weinstein (1988) for another point of view regarding the connection in this case.

Our paper is devoted to demonstrating result (B), and its consequences. Result (A), especially its dynamical interpretations and consequences, will be the subject of a future paper with Marsden and Ratiu (1988). The author is indebted to Marsden and Ratiu, who felt strongly that integrability must be a spurious assumption in any nice geometric framework for the Hannay angles. The author would not have stumbled across (A) without their prodding.

The uniqueness of the connection satisfying the three axioms lets us obtain an alternate formula, (3.3), for it. This alternative formula becomes especially simple, Eq. (3.5), when there is an additional symmetry group present which also acts on parameter space. This same type of extra symmetry has been used in the quantum case by several authors to explicitly calculate the quantum connection. Among these are Gerbert (1987), Jackiw (1987), Anandan (1988), and Vinet (1988).

Equation (3.5) is used to compute the connection and its holonomy for two examples. The first (Sect.4) is the Foucault pendulum, and non-integrable 
generalizations of it, Examples $1 \mathrm{a}$ and $\mathrm{b}$. The parameter space is a two-sphere. The Hannay-Berry connection turns out to be essentially the standard Levi-Civita connection on the sphere. This result was stated without proof by Hannay (1985). Koiller (1987b), gave a proof. This paper began as an attempt to geometrize and extend Koiller's calculations.

The second example (Sect. 6) is a family of linear oscillators. The parameter space is the set of positive definite matrices with nondegenerate eigenvalues. We find that the curvature of the Hannay-Berry connection to be zero, but the holonomy turns out to be non-trivial. Berry obtained these same results in his original (1984) paper.

\section{Families of Hamiltonian Group Actions}

Let $P$ be a symplectic manifold with symplectic form $\omega$. A family of Hamiltonian $G$-actions on $P$ is a smooth action of the Lie group $G$ on $P \times M$, where $M$ is a manifold which we call parameter space, and where

(1) each fiber $P \times\{x\}, x \in M$, is invariant under the action,

(2) the action restricted to each fiber is symplectic,

(3) the action admits a smooth family of moment maps $I: P \times M \rightarrow \mathfrak{g}^{*},(\mathfrak{g}$ denotes the Lie algebra of $G, \mathfrak{g}^{*}$ its dual).

This last property means that for each $\lambda \in \mathfrak{g}$ the corresponding infinitesimal generator $\lambda_{P \times M}$ for the action is equal to the Hamiltonian vector field generated by the real-valued function $I^{\lambda}=\langle I, \lambda\rangle$ on $P \times M$. This is an equality of "vertical" vector fields on $P \times M$, where "vertical" means tangent to the fibres. In order to fix notation we will be more explicit

where

$$
\lambda_{P \times M}=X_{I^{\lambda}},
$$

$$
\lambda_{P \times M}(p, x)=d(\exp (s \lambda) \cdot(p, x)) /\left.d s\right|_{s=0} .
$$

And for $f$ a real valued function on $P \times M$ its Hamiltonian vector field, $X_{f}$ is defined by

$$
\omega \oplus 0\left(X_{f}, \cdot\right)=d_{P} f,
$$

where $\omega \oplus 0$ denotes the pull-back of $\omega$ to $P \times M$ by the projection onto $P$, and where $d_{P}$ denotes exterior derivative in the $P$-direction. If we fix canonical parameter-independent coordinates $\left\{\left(q^{i}, p_{i}\right)\right\}$ on $P$ and coordinates $\left\{x^{a}\right\}$ on $M$ then

$$
\omega \oplus 0=\sum d q^{i} \wedge d p_{i} \quad \text { and } \quad X_{f}=\sum\left\{\left(\partial f / \partial p_{i}\right) \partial / \partial q^{i}-\left(\partial f / \partial q^{i}\right) \partial / \partial p_{i}\right\} \text {. }
$$

Note that if $G$ is connected then all the information in the Hamiltonian family is contained in the family of moment maps $I: P \times M \rightarrow \mathrm{g}^{*}$. When there will be no confusion, we will simply refer to $I$ as the moment map.

Example 1a. Here $G$ will be the circle $S^{1}$. Suppose the rotation group $S O(3)$ acts in a Hamiltonian fashion on $P$ with equivariant moment map $J: P \rightarrow s o(3)^{*}=\mathbb{R}^{3}$. $(J$ is the angular momentum vector of the system.) Set $M=S^{2} \subset \mathbb{R}^{3}$, a sphere of radius $r$ centered at the origin. Identify $\mathbb{R}^{3}$ with the Lie algebra $s o(3)$ in the standard fashion. Define

$$
I(p, x)=\langle J(p), x\rangle \text {. }
$$


For each $x \in S^{2}$, the Hamiltonian flow of $I(\cdot, x)$ defines an $S^{1}$ action on $P$. By having $S^{1}$ act trivially on $S^{2}$ we thus obtain an $S^{1}$ action on $P \times M$, and hence a family of Hamiltonian $S^{1}$ actions on $P$.

Here is an alternative description of this action. First, for each $x$ in $S^{2}$, embed $S^{1}$ into $S O(3)$ by the homomorphism $i_{x}: S^{1} \rightarrow S O(3)$ obtained by thinking of $S^{1}$ as the set of (counterclockwise) rotations about the $x$-axis. Second, compose $i_{x}$ with the given $S O(3)$ action on $P$.

Example $1 b$ generalizes the previous example by replacing $S O(3)$ with a compact Lie group $G^{1} \supset G$ acting in a Hamiltonian fashion on $P$, with equivariant moment map $J: P \rightarrow \mathfrak{g}^{1 *}$, and by replacing $S^{1}$ by $G$, a maximal torus in $G^{1}$. We will identify $G$ with the isotropy group, $G_{\mu}^{1}$, of a fixed regular element $\mu \in \mathfrak{g}^{1 *}$. Thus $G$ is a maximal torus. The parameter space $M=G_{1} / G$ is the co-adjoint orbit through $\mu$. The family of moment maps is defined by

$$
\langle I(p, x), \lambda\rangle=\left\langle J(p), i_{x} \lambda\right\rangle, \quad \lambda \in \mathfrak{g},
$$

where $i_{x}: \mathfrak{g}_{\mu}^{1} \rightarrow \mathfrak{g}_{x}^{1} \rightarrow \mathfrak{g}^{1}$ is the linear map formed by composing the inclusion $\mathfrak{g}_{x}^{1} \rightarrow \mathfrak{g}^{1}$ with the isomorphism $\mathfrak{g}_{\mu}^{1} \rightarrow \mathfrak{g}_{x}^{1}$, defined by $\xi \mapsto \mathrm{Ad}_{g} \xi$, where $x=\left(\operatorname{Ad}_{g}\right)^{-1 *} \mu$. (We need $G$ abelian in order for this map to be independent of the choice of $g$.)

Example 2. A family of completely integrable systems consists of a function $H: P$ $\times M \rightarrow \mathbb{R}$ such that for each $x_{0}$ in $M, H\left(\cdot, x_{0}\right)$ is completely integrable on $P$.

Recall this means that $H\left(\cdot, x_{0}\right)$ can be written as a function of $n=\frac{1}{2} \operatorname{dim} P$ smooth functions $f_{1}\left(\cdot, x_{0}\right), f_{2}\left(\cdot, x_{0}\right), \ldots, f_{n}\left(\cdot, x_{0}\right)$ on $P$, called "integrals in involution," whose Poisson brackets with each other are all zero, and which are independent $\left(d f_{1}\left(\cdot, x_{0}\right) \wedge d f_{2}\left(\cdot, x_{0}\right) \wedge \ldots \wedge d f_{n}\left(\cdot, x_{0}\right) \neq 0\right)$ almost everywhere. The fact that their Poisson brackets are zero implies that the flows of their Hamiltonian vector fields commute. A set of action variables $I\left(\cdot, x_{0}\right)=\left(I_{1}\left(\cdot, x_{0}\right), I_{2}\left(\cdot, x_{0}\right), \ldots\right.$, $\left.I_{n}\left(\cdot, x_{0}\right)\right)$ for $H\left(\cdot, x_{0}\right)$ is a set of integrals in involution such that the flow of each Hamiltonian vector field is $2 \pi$-periodic. Action variables always exist locally in a neighborhood of a point where the common level sets of the $f_{i}\left(\cdot, x_{0}\right)$ are compact.

Suppose that the family of completely integrable systems admits global smooth action variables $I=\left(I_{1}, I_{2}, \ldots, I_{n}\right): P \times M \rightarrow \mathbb{R}^{n}, n=\frac{1}{2} \operatorname{dim} P$. Then $I$ can be viewed as a family of moment maps for a family of Hamiltonian $G$-actions, where $G$ is the $n$-torus and $\mathbb{R}^{n}$ its dual Lie algebra. The case where $H$ does not admit global action variable will be discussed in Sect. 5 .

What makes a family of Hamiltonian $G$-actions interesting is that the $G$ action can depend on $M$. Infinitesimal stated, this means that the Lie bracket $\left[\lambda_{P \times M}, 0 \oplus V\right]$ is not zero [see (1.1)]. Here $\lambda \in \mathfrak{g}, V$ is a vector field on $M$, and $0 \oplus V$ denotes its obvious extension to a vector field on $P \times M$. The Hannay-Berry connection will provide a measure of the extent to which the action depends on the parameters.

Notation. We will often abuse notation by making the identifications $0 \oplus V=V$, $\omega \oplus 0=\omega$.

One calculates

$$
\left[\lambda_{P \times M}, V\right]=X_{-d I^{\lambda \cdot V}}, \quad V \text { a vector field on } M .
$$


This formula is key to the proof of our main result, Theorem 2. To prove (1.1), write $V=\partial_{1}$, thought of as a differential operator, and apply the Lie bracket to a function $f:$

$$
\begin{aligned}
{\left[\lambda_{P \times M}, \partial_{1}\right][f] } & =\lambda_{P \times M}\left[\partial_{1} f\right]-\partial_{1}\left[\lambda_{P \times M}[f]\right] \\
& =\left\{\partial_{1} f, I^{\lambda}\right\}-\partial_{1}\left\{f, I^{\lambda}\right\} \\
& =\left\{\partial_{1} f, I^{\lambda}\right\}-\left\{\partial_{1} f, I^{\lambda}\right\}-\left\{f, \partial_{1} I^{\lambda}\right\}=\left\{f,-\partial_{1} I^{\lambda}\right\},
\end{aligned}
$$

where $\{\cdot, \cdot\}$ denote Poisson brackets on $P$.

\section{Defining the Connection and Relating it to Hannay}

\subsection{Defining the Connection}

From now on assume that $G$ is compact and connected. Then we can average with respect to Haar measure, $d g$, on $G$. If $\alpha$ is any tensor field defined along (not on) a $G$-invariant submanifold of $P \times M$ we can form its average

$$
\langle\alpha\rangle=\int_{G}\left(g^{*} \alpha\right) d g / \operatorname{vol}(G),
$$

which is now a $G$-invariant tensor field.

Definition. The horizontal lift $h \cdot v$ of a vector $v \in T_{x} M$ with respect to the HannayBerry connection is the vector field

$$
h \cdot v=\langle 0 \oplus v\rangle
$$

along $P \times\{x\}$. Here $0 \oplus v$ denotes the vector field $p \mapsto(0, v) \in T_{p} P \times T_{x} M$ along $P \times\{x\} \subset P \times M$.

This defines the horizontal lift for a connection in the sense of Ehresmann on the bundle $\pi: P \times M \rightarrow M$. (At the beginning of Sect. 3 we recall the definition of an Ehresmann connection in case the reader is unfamiliar with it.) A coordinate expression may be helpful. Take the case $P=\mathbb{R}^{2}$ with coordinates $(q, p), M=\mathbb{R}$ with coordinate $x$, and $G=S^{1}$. Let $R_{\theta}$ denote the action of $\theta \in S_{1}$. In coordinates

$$
R_{\theta}(q, p, x)=(Q(q, p, \theta, x), P(q, p, \theta, x), x) .
$$

Then $R_{\theta}^{*} \partial / \partial x=\partial / \partial x+(\partial Q / \partial x) \partial / \partial q+(\partial P / \partial x) \partial / \partial p$, so that

$$
h \cdot v=\partial / \partial x+\langle(\partial Q / \partial x)\rangle \partial / \partial q+\langle(\partial P / \partial x)\rangle \partial / \partial p .
$$

Remark. If the $G$-action is independent of $M$ [i.e. (1.1) is zero] then $g^{*}(0 \oplus v)=0 \oplus v$. Consequently $h \cdot v=0 \oplus v$, hence (2.1) defines the trivial connection.

The Hannay-Berry connection was motivated by dynamical considerations. A function $H: P \times M \rightarrow \mathbb{R}$ together with a curve $\gamma: \mathbb{R} \rightarrow M$, define a time-dependent Hamiltonian system on $P$, namely that given by the time-dependent Hamiltonian $H_{\gamma}(p, t)=H(p, \gamma(t))$ on $P$. This dynamical system is the flow of the vector field $X_{H} \oplus \dot{\gamma}$ on $P \times \operatorname{im} \gamma$, where $\dot{\gamma}=d \gamma / d t$.

Suppose we believe that the "group variables" $G$ (i.e. coordinates on the $G$-orbits) are "fast" relative to the "other variables" (coordinates on $P \times M / G$ ). 
Then, at least in the case $G$ Abelian, the averaging principle [see, for example, Arnol'd (1978)] suggests that it is a good approximation to replace $X_{H} \oplus \dot{\gamma}$ with its average $\left\langle X_{H}+\dot{\gamma}\right\rangle=X_{\langle H\rangle}+\langle\dot{\gamma}\rangle$. If $H$ is $G$-invariant then $\langle H\rangle=H$. Thus the averaging principle says to

$$
\text { "replace } X_{H}+\dot{\gamma} \text { with } X_{H}+h \cdot \dot{\gamma} \text {. }
$$

\subsection{Relation to Hannay}

Suppose we are in the context of Example 2, so that $H$ is a family of completely integrable systems and $I$ consists of global actions variables. Consider the sets

$$
E_{x}^{\mu}:=I^{-1}(\mu) \cap P \times\{x\},
$$

where $\mu \in \mathbb{R}^{n}$ is a regular value of $I(\cdot, x), x$ a fixed parameter. These consist of a disjoint union of $n$-tori (Arnold-Liousville theorem). In a neighborhood $U \subset P \times M$ of any one such torus there exist local angle variables

$$
\theta=\left(\theta^{1}, \theta^{2}, \ldots, \theta^{n}\right): U \subset P \times M \rightarrow T^{n}, \quad \text { the } n \text {-torus . }
$$

These are obtained by choosing a local (Lagrangian) slice, i.e. choice of " $\theta=0$ ", for the toral action, then pushing this around by the toral action. $\theta(\cdot, x)$ coordinatizes our original torus which is a component of $E_{x}^{\mu}$. For $x^{\prime}$ near $x$ and $\mu^{\prime}$ near $\mu, E_{x^{\prime}}^{\mu^{\prime}}$ is also a disjoint union of $n$-tori, and the component nearest our original torus is coordinatized by $\theta\left(\cdot x^{\prime}\right)$.

The action-angle variables $(I, \theta)$ form a parameter-dependent coordinate system on $P$. Then $\left\{I^{i}, \theta_{i}, x^{a}\right\}$ form coordinates on $P \times M$, where the $x^{a}$ are fixed coordinates on $M$, as above. In these coordinates

$$
0 \oplus \partial / \partial x^{a}=\Sigma\left(\partial I^{i} / \partial x^{a}\right) \partial / \partial I+\Sigma\left(\partial \theta^{i} / \partial x^{a}\right) \partial / \partial \theta+\partial / \partial x^{a}
$$

and

$$
X_{H}=\Sigma \omega^{i}(I, x) \partial / \partial \theta^{i},
$$

where $\omega^{i}=\partial H / \partial I^{i}$.

It is well-known that the standard actions $I$ (see Sect. 5 for their definition) satisfy $\left\langle\partial I^{i} / \partial x^{a}\right\rangle=0$, or, in more invariant notation,

$$
\left\langle d_{M} I\right\rangle=0, \quad I \text { standard } .
$$

Here $d_{M}$ denotes exterior differentiation with respect to $M$. In coordinates $\left\{x^{a}\right\}$ on $M$ :

$$
d_{M} I=\Sigma\left(\partial I / \partial x^{a}\right) d x^{a}, \quad \text { a one-form on } P \times M .
$$

Warning: Most actions do not satisfy (2.3), for if $I$ is an action, (i.e. generates the given toral action), then so is $I+f$, where $f$ is a function on $M$. But $\left\langle d_{M}(I+f)\right\rangle$ $=\left\langle d_{M} I\right\rangle+d f$.

Remark. Equation (2.3) is one of the basic facts regarding slowly varying completely integrable systems. A proof using generating functions can be put together from Arnold (1978). Alternatively, see Weinstein (1988), or write the author. 
For slowly varying one degree of freedom systems (2.3) implies that the standard actions are adiabatic invariants (Arnold, 1978, 1983). This means that on a time scale of order $1 / \varepsilon$, where $\varepsilon$ measures how slowly the system varies, the actions vary by at most $\varepsilon$. This is proved by using the $S^{1}$ averaging theorem. Due to resonances, a corresponding toral averaging theorem is no longer true for more than one degree of freedom, so that the standard actions are not in general adiabatic invariants, even though (2.3) holds (see Arnold, 1983).

In any case, if the actions are standard

$$
h \cdot \partial / \partial x^{a}=\Sigma\left\langle\partial \theta^{i} / \partial x^{a}\right\rangle \partial / \partial \theta^{i}+\partial / \partial x^{a},
$$

and so for $\gamma$ a curve in $M$

$$
h \cdot \dot{\gamma}=\Sigma\left(\left\langle d_{M} \theta^{i}\right\rangle \cdot \dot{\gamma}\right) \partial / \partial \theta^{i} \oplus \dot{\gamma}, \quad \text { where } \dot{\gamma}=d \gamma / d t .
$$

The equations for parallel translation with respect to the Hannay-Berry connection are then

$$
d \theta^{i} / d t=\left\langle d_{M} \theta^{i}\right\rangle \cdot \dot{\gamma}, \quad d I^{i} / d t=0 .
$$

So that

$$
\theta(t)=\theta_{0}+\int_{\gamma}\left\langle d_{M} \theta\right\rangle,
$$

where $\left\langle d_{M} \theta\right\rangle$ is one-form with values in the Lie algebra of the torus. Hannay (1985) defined

$$
\text { Hannay's angles: }=\int_{\gamma}\left\langle d_{M} \theta\right\rangle \text {. }
$$

Thus, in the case where $\gamma$ is a loop contained in our coordinate patch,

Hannay's angles = the holonomy of the Hannay-Berry connection .

[Usually the integral (2.4) is rewritten, using Stoke's formula, as the integral of a two-form over a disc bounding $\gamma$.] We summarize and extend this discussion in:

Theorem 1. Suppose that $I$ is a set of global action variables for a family of completely integrable systems, and that $\left\langle d_{M} I\right\rangle=0$. Then for small enough loops the holonomy of the Hannay-Berry connection is the Hannay angles. Parallel translation with respect to the connection leaves invariant the submanifolds

$$
E^{\mu}=\left\{(p, x): I(p, x)=\mu \in \mathbb{R}^{n}\right\} \subset P \times M,
$$

and hence induces a connection on them. The connection's curvature is given by the formula of Theorem 2.

Suppose that $M$ is connected. Also suppose that $\mu$ is such that the fibers $E_{x}^{\mu}$ of $E^{\mu}$ are connected, and that for one parameter value $x_{0} \in M, \mu$ is a regular value of $I\left(\cdot, x_{0}\right)$. Then

(1) $E^{\mu} \rightarrow M$ is a principal torus bundle,

(2) the Hannay-Berry connection, restricted to it, is a connection in the principal bundle sense.

Proof. The horizontal lift of a vector with respect to the Hannay-Berry connection is manifestly invariant under the torus action. Hence if $E^{\mu}$ is a principal bundle then 
the connection is a principal bundle connection. In order to show that $E^{\mu}$ is a principal torus bundle, note that by the connectivity assumption $E_{x_{0}}^{\mu}$ is an $n$-dimensional orbit of the torus action, hence an $n$-torus. The same follows for any $E_{x}^{\mu}$ as can be seen by connecting $x_{0}$ to $x$ by a smooth path $\gamma$ and then parallel translating along $\gamma$. (Parallel translation restricted to $E^{\mu}$ is complete since the fibers of $E^{\mu}$ are compact.) This defines a diffeomorphism from $E_{x_{0}}^{\mu}$ to $E_{x}^{\mu}$ which intertwines the toral actions. It follows that $E^{\mu}$ is a principal bundle. (It may be that the action of the torus $G$ on $E^{\mu}$ has a finite nontrivial isotropy group $\Gamma$, in which case the action of $G / \Gamma$ is free, and $G / \Gamma$ is the correct structure group for the bundle.)

Remark 1. The connectivity of the fiber $E_{x}^{\mu}$ is automatic when $P$ is compact. This was proved by Atiyah (1982) and Guillemin-Sternberg (1984) in their papers on the convexity of the image of $I$.

Remark 2. In Sect. 5 we extend Theorem 1 to families of completely integrable systems which do not admit global actions.

\subsection{Dynamical Interpretation of the Holonomy: \\ Integrable Versus Non-Integrable Case}

Let $\gamma$ be a loop in $M$ based at $x_{0}$. Roughly speaking, the holonomy of the HannayBerry connection around $\gamma$ provides a way of comparing the dynamics for the timeindependent vector field $X_{H}$ obtained by freezing the parameter value at $x_{0}$ (setting $\dot{\gamma}=0$ ) to the time-dependent dynamics, $X_{H}+\dot{\gamma}$. This is not quite correct, since the dynamics in the fiber will in general vary as we move along $\gamma$ in parameter space. In the integrable case this fiber dynamics is easily accounted for as a "dynamic phase"

$$
\Delta \theta_{\mathrm{dyn}}^{i}=\int \omega^{i}(I, \gamma(t)) d t,
$$

so that, in the averaging approximation the total angular shift upon going around the loop is

$$
\Delta \theta=\Delta \theta_{\mathrm{dyn}}+\text { Hannay's angles . }
$$

This assumes, of course, that we return to the torus we started on, which is automatic if the loop is small enough, or if the hypothesis of the second part of Theorem 1 are satisfied.

The fiber dynamics cannot be so easily accounted for in the non-integrable case. Qualitatively new phenomena appear which have to do with the bundle of symplectic reduced spaces

$$
I^{-1}(\mu) / G_{\mu} \rightarrow M .
$$

These phenomena will be the subject of a future paper with Marsden and Ratiu. See Sect. 8 for more information.

In writing (2.5) we are assuming that $I$ is equivariant. $G_{\mu}$ denotes the isotropy group of $\mu \in \mathfrak{g}^{*}$. The bundle (2.5) is sandwiched between $I^{-1}(\mu)$ and $M$ :

$$
I^{-1}(\mu) \rightarrow I^{-1}(\mu) / G_{\mu} \rightarrow M .
$$

Its fiber over $M$ is the symplectic reduced space of Marsden and Weinstein (1974). The dynamics on these fibers will in general be non-integrable, perhaps ergodic, or 
chaotic. In the completely integrable case the fiber is a discrete point set and the $I^{-1}(\mu)$ fiber-dynamics is specified by vectors attached to these points, namely the frequency vectors $\omega=\left(\omega^{1}, \omega^{2}, \ldots, \omega^{n}\right)$.

\section{Main Results: Geometric Properties of the Connection}

\subsection{Hamiltonian Connections and the Main Result}

To begin our discussion of the geometric properties of the Hannay-Berry connection, (2.1), we recall some definitions regarding Ehresmann connections. A connection in the sense of Ehresmann on the bundle $\pi: P \times M \rightarrow M$ is a smooth distribution Hor on $P \times M$, called the horizontal distribution, such that $T P \oplus$ Hor $=T(P \times M)$. Equivalently, it is a smooth family of linear endomorphisms hor $_{p, x}: T_{x} M \rightarrow T_{p} P \times T_{x} M$, called horizontal lifts, satisfying $d \pi_{p, x} \circ$ hor $_{p, x}=$ identity on $T_{x} M$. Note we can always write $\operatorname{hor}_{p, x} \cdot v=Y(p, x) \cdot v \oplus v$, where $Y(p, x): T_{x} M \rightarrow T_{p} P$ is linear. Parallel translation along a path $\gamma$ in $M$ is the (local) flow defined by the vector field hor $\circ d \gamma / d t$ on $P \times \operatorname{im} \gamma$. The covariant derivative of a function $f$ on $P \times M$ at $x$ is given by $D f \cdot v=d f \cdot(h \cdot v)$, a function on $P \times\{x\}$. Df is a one-form on $M$ with values in the ring of functions on $P \times M$.

Definition. A Hamiltonian connection for the family of Hamiltonian $G$-actions $I: P \times M \rightarrow \mathrm{g}^{*}$ is an Ehresmann connection on $P \times M \rightarrow M$ which satisfies the following three axioms:

(1) parallel translation preserves the level sets of $I$,

(2) parallel translation is Hamiltonian,

(3) for each $v \in T_{x} M$ the average of the corresponding Hamiltonian function $K \cdot v$ is a constant on $P \times\{x\}$. Without loss of generality we will take this constant to be zero.

Explanation. (1) is equivalent to $D I=0$.

(2) Means that for each $v \in T_{x} M$ there is a function $K \cdot v$ on $P$ such that

$$
\text { hor } \cdot v=X_{K \cdot v} \oplus v \text {. }
$$

Thus parallel translation is given by the (local) flow of a time-dependent Hamiltonian vector field. Note that the connection is uniquely determined by the map

$$
v \mapsto K \cdot v
$$

considered as a one-form on $M$ with values in $C^{\infty}(P \times M) / C^{\infty}(M)$. We will call $K$ the Hamiltonian one-form of the connection. Note that in general $K \cdot v$ is not $G$-invariant (since $0 \oplus v$ is not).

(3) Is a normalization condition: $\langle K \cdot v\rangle=0$.

There can be at most one Hamiltonian connection. For suppose we have two Hamiltonian connections with horizontal lifts denoted $h_{1}, h_{2}$ and Hamiltonian one-forms denoted $K_{1}, K_{2}$. Then $h_{1} \cdot v-h_{2} \cdot v=X_{K_{1} \cdot v-K_{2} \cdot v}$, so by property (1) $d I \cdot X_{K_{1} \cdot v-K_{2} \cdot v}=0$. For each $\lambda \in \mathfrak{g}, d I^{\lambda} \cdot X_{K_{1} \cdot v-K_{2} \cdot v}=\lambda_{P \times M}\left[K_{1} \cdot v-K_{2} \cdot v\right]$, therefore $K_{1} \cdot v-K_{2} \cdot v$ is $G$-invariant, since $G$ is connected. Consequently 
$\left\langle K_{1} \cdot v-K_{2} \cdot v\right\rangle=K_{1} \cdot v-K_{2} \cdot v$. But $\left\langle K_{1} \cdot v-K_{2} \cdot v\right\rangle=\left\langle K_{1} \cdot v\right\rangle-\left\langle K_{2} \cdot v\right\rangle=0$ by the normalization property (3). Consequently $K_{1}=K_{2}$ and the two connections are equal.

Our main result, Theorem 2, states that under the hypothesis

$$
\left\langle d_{M} I\right\rangle=0
$$

that the Hamiltonian connection exists, and is equal to the Hannay-Berry connection. Equation (3.2) is a necessary condition for the existence of a connection satisfying (1) and (2). For then

$$
0=D I^{\lambda} \cdot v=d_{M} I^{\lambda} \cdot v+d_{P} I^{\lambda} \cdot \xi_{K \cdot v}=d_{M} I^{\lambda} \cdot v-\lambda_{P \times M}[K \cdot v] .
$$

The second term is the derivative of function with respect to an infinitesimal generator, and hence has average zero, since $G$ is compact. (Think of the case $G=S^{1}$, in which case this term is $\partial K \cdot v / \partial \theta$.) Averaging this equation then yields (3.2).

Theorem 2. Suppose that $\left\langle d_{M} I\right\rangle=0$, where I is the moment map for a family of Hamiltonian $G$-actions on $P$, and $G$ is a compact connected Lie group. Then there is $a$ unique Hamiltonian connection on $P \times M$. It equals the Hannay-Berry connection, as defined by the averaging formula (2.1):

$$
h \cdot v=\langle 0 \oplus v\rangle .
$$

This can also be written

$$
h \cdot v=X_{K \cdot v}+v
$$

where the Hamiltonian one-form $K$ satisfies defining axioms (1), (2), and (3) above. Its horizontal distribution is

$$
\text { Hor }_{p, x}=\left\langle 0 \oplus T_{x} M\right\rangle_{p, x},
$$

a G-invariant distribution. Its covariant differentiation operator is given by

$$
D f \cdot v=d f \cdot v+\{f, K \cdot v\}
$$

on functions, and satisfies

$$
D\langle\alpha\rangle=\langle D \alpha\rangle=\langle d \alpha\rangle \circ n
$$

for any function or $k$-form $\alpha$ on $P \times M$. The curvature of the connection is

$$
\Omega=\langle\omega \oplus 0\rangle \circ h,
$$

or equivalently

$$
\Omega\left(v_{1}, v_{2}\right)=\left\langle\left\{K \cdot v_{1}, K \cdot v_{2}\right\}\right\rangle
$$

Proof. See Sect. 7.

Explanation of Curvature. View $P \times M \rightarrow M$ as a symplectic fiber bundle. [See Gotay et al. (1980) for a treatment of symplectic fiber bundles.] If we want to preserve the foliation of the total space given by the level sets of $I$, then the current structure group for this bundle is the group of symplectic transformations of 
$P \times\{x\}$ which commute with the $G$-action on $P \times\{x\}$. (Note that the action of $G$ defines a natural homomorphism of $G$ into this structure group.) The Lie algebra of this structure group is the algebra $C_{G}^{\infty}(P \times\{x\})$ of $G$-invariant functions on $P \times\{x\}$ (modulo constants) under Poisson bracket. Our formulas for the curvature $\Omega$ are formulas for 2-forms on $M$ with values in the ring of $C_{G}^{\infty}(P \times M)$ of $G$-invariant functions.

Hamiltonian Connection as Induced Connection. In view of axiom (1), DI =0, the Hannay-Berry connection induces a connection on any level set of $I$. In the definition (2.1) of the connection, averaging acts like a projection operator applied to the trivial connection. In these ways the Hannay-Berry connection is analogous to the induced Levi-Civita connection of a submanifold embedded in a Euclidean space.

\subsection{Regarding the Hypothesis $\left\langle d_{M} I\right\rangle=0$}

Proposition 1. Suppose I: $P \times M \rightarrow \mathrm{g}^{*}$ is the moment map for a family of Hamiltonian G-actions on $P, G$ compact and connected. Then $\left\langle d_{M} I\right\rangle$ is the pull-back of a closed one-form on $M$.

We prove this in Sect. 7.

Corollary. In a neighborhood of any point $x_{0} \in M$ there is a $\mathfrak{g}^{*}$-valued function $f$ on $M$ such that $I^{\prime}=I+f \circ \pi$ satisfies $\left\langle d_{M} I^{\prime}\right\rangle=0$.

Proof of Corollary.

$$
f(x)=\int_{x_{0}}^{x}\left\langle d_{M} I\right\rangle,
$$

where by abuse of notation we write $\left\langle d_{M} I\right\rangle$ for the one-form whose pull-back is $\left\langle d_{M} I\right\rangle$.

Note that $I$ and $I^{\prime}$ generate the same $g$-action, since $f$ is constant on $P \times\{x\}$. Thus the $G$-action on $P \times M$ is unaltered by the replacement of $I$ by $I^{\prime}$. Reworded then, the corollary states that

it is always possible to change the moment map $I$,

without changing the $G$-action, in such a way that $\left\langle d_{M} I\right\rangle=0$.

This new choice of $I$ is global in $P$, but local in $M$. It is always possible to make the choice global if either

(a) $M$ is simply connected,

(b) we allow $I$ to be multiple valued.

\subsection{An Alternative Formula for the Connection}

For $v \in T_{x} M$, suppose that we have found a smooth function $\tilde{K} \cdot v$ on $P \times\{x\}$ satisfying

$$
d I^{\lambda} \cdot v+\left\{I^{\lambda}, \tilde{K} \cdot v\right\}=0
$$


for all $\lambda$ in $\mathfrak{g}^{*}$. [Essentially the same formula appears in Anandan (1988) as Eq. (13).] Then

$$
K \cdot v=\tilde{K} \cdot v-\langle\tilde{K} \cdot v\rangle
$$

defines the Hamiltonian one-form for the Hannay-Berry connection. This is easily seen by checking the three defining axioms for a Hamiltonian connection, and invoking uniqueness.

Definition. We say that a function $\tilde{K} \cdot v$ satisfying (3.3a) almost generates parallel translation in the direction $v$.

\subsection{Formula for the Connection in the Presence of Additional Symmetry}

Suppose another Lie group $G^{1}$ (typically containing $G$ ) acts separately on $P$ and on $M$ in such a way that the diagonal action on $P \times M$ leaves the moment map $I: P \times M \rightarrow \mathrm{g}^{*}$ invariant:

$$
I(g p, g x)=I(p, x)
$$

Also assume that the $G_{1}$ action on $P$ is Hamiltonian, with equivariant moment $\operatorname{map} J: P \rightarrow \mathfrak{g}^{1 *}$.

Definition. We call a group $G^{1}$ together with such a diagonal action a symmetry group of the family of Hamiltonian systems.

Differentiating Eq. (3.4) of invariance with respect to $g$ we obtain Eq. (3.3a):

$$
\left\{I, J^{\xi}\right\}+d I \cdot \xi_{M}=0 \text { for } \xi \in \mathfrak{g}_{1} .
$$

Here $J^{\xi}=\langle J, \xi\rangle$ denotes the $\xi$-component of $J$, and $\xi_{M}$ denotes the infinitesimal generator on $M$ corresponding to $\xi$. It follows [Eq. (3.3b)] that the Hamiltonian function for the horizontal lift of $\xi_{M}$ is

$$
K \cdot \xi_{M}=J^{\xi}-\left\langle J^{\xi}\right\rangle .
$$

If $G^{1}$ acts transitively on $M$ this determines the connection. In the next section we will use (3.5) to calculate the connection for our first examples.

\section{Some Examples, Including the Foucault Pendulum}

Example $1 a$ (see Sect. 1). Equivariance of $J$ means that $J(g \cdot p)=g \cdot J(p)$, for $g \in S O(3)$. The invariance, (3.4), of easily follows. We can now calculate the connection by using formula (3.5).

Fix the parameter $x \in S^{2}$. Let $\left\{e_{1}, e_{2}, e_{3}\right\}$ be an oriented orthonormal basis for $\mathbb{R}^{3}$, with $e_{3}=x / r$, where $r$ is the radius of the sphere. Set $J_{i}=\left\langle J, e_{i}\right\rangle, i=1,2,3$. Then $I(p, x)=r J_{3}$. Let $R_{\theta}$ denote the action of the group $G=S^{1}$ on the fiber $P \times\{x\}$. We have $R_{\theta} p=\exp \theta e_{3} \cdot p$. By equivariance again,

$$
J_{1}\left(R_{\theta} p\right)=\cos \theta J_{1}(p)+\sin \theta J_{2}(p)
$$

with a similar formula for $J_{2}$. It follows that $\left\langle J_{1}\right\rangle=\left\langle J_{2}\right\rangle=0$. Also $\left\langle J_{3}\right\rangle=J_{3}$. 
Remembering the identification of $s o(3)$ with $\mathbb{R}^{3}$, we note that the infinitesimal generators are given by $\xi_{S_{2}}(x)=\xi \times x$. So at $x=r e_{3},\left(e_{1}\right)_{S^{2}}=-r e_{2},\left(e_{2}\right)_{S^{2}}=r e_{1}$.

Thus $K \cdot e_{1}=J_{2} / r$, and $K \cdot e_{2}=-J_{1} / r$. $K$ can also be expressed by $K \cdot v=\langle J$, $v \times x\rangle / r$, or

$$
K \cdot \xi_{S^{2}}(x)=\left\langle J, \mathbb{P}_{x} \xi\right\rangle,
$$

where $\mathbb{P}_{x}$ denotes the orthogonal projection onto the plane tangent to $S^{2}$ at $x$.

The curvature is then given by

$$
\Omega\left(e_{1}, e_{2}\right)=\left\langle\left\{J_{2},-J_{1}\right\}\right\rangle / r^{2}=\left\langle J_{3}\right\rangle / r^{2}=I / r^{2} .
$$

Rember this is to be interpreted as a vertical vector field by taking its Hamiltonian vector field. The Hamiltonian vector field corresponding to $I$ is $\partial / \partial \theta$, the infinitesimal generator of the $S^{1}$ action on $P \times M$. Thus we can write $\Omega=\partial / \partial \theta \otimes r^{-2} d A$, where $d A$ is the standard area form on the sphere. Note that $r^{-2} d A$ is the curvature form of the Levi-Civita connection on our sphere. Consequently, the holonomy about a loop (based at $x$ ) is $p \rightarrow R_{\Delta \theta} \cdot p$, where

$$
\Delta \theta=\text { area of the solid angle subtended by the curve. }
$$

Foucault Pendulum (see Fig. 1). The holonomy (4.1) has been invoked by Koiller (1987), and Hannay (1985) as providing an alternative derivation of the precession of the Foucault pendulum. We will show below that this derivation is not justified, because the $S^{1}$ averaging cannot be. But it does give the correct answer, so let us go through it regardless.

The Foucault pendulum is a spherical pendulum hanging at a latitude $\alpha$ above the earth's surface. In the course of one day the pendulum precesses by an amount

$$
\Delta \theta=-2 \pi \cos \alpha .
$$

Modulo $2 \pi$, this equals $2 \pi(1-\cos \alpha)$ which is the spherical area inside the circle of lattitude $\alpha$, hence the holonomy of the Hannay-Berry connection. This precession is measured in a "lab frame" sitting on the earth near the pendulum and can be attributed to the Coriolis force.

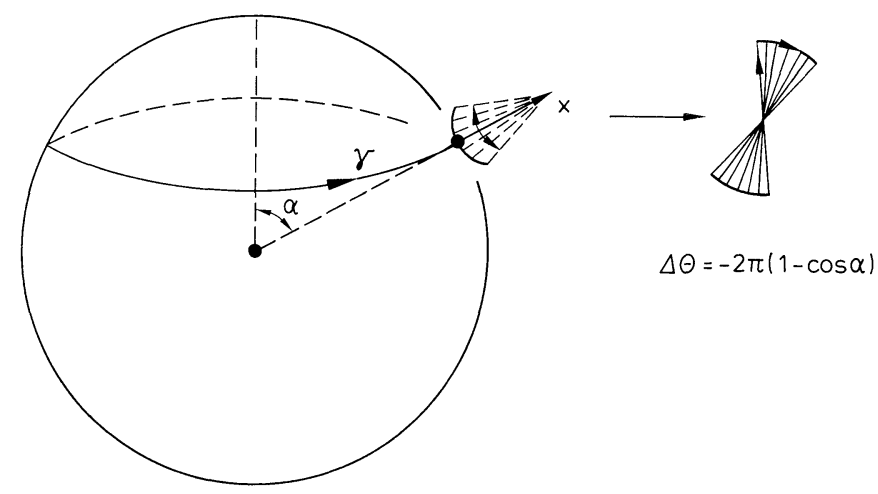

Fig. 1. Foucault pendulum 
In the lab frame the pendulum's position is described by a point $q_{L}$ on a sphere with radius equal to the length of the pendulum bob. The coordinates of the pendulum in the lab frame are related to those of an inertial frame, $Q$, centered at the earth's center, by $Q=R_{t}\left(q_{L}+r_{0}\right)$. Here $R_{t}=\exp t \Omega$ is the rotation matrix describing the earth's rotation and $r_{0}$ is the position vector of the pendulum's suspension point at some initial time. If instead we use coordinates $q \in S^{2}$ given by $q=R_{t} q_{L}$, we find that the Hamiltonian of the Foucault pendulum is

$$
H_{\alpha}: T^{*} S^{2} \times S^{1} \rightarrow \mathbb{R} ; \quad H_{\alpha}(q, p ; t)=\frac{1}{2 m}\|p\|^{2}+m g\left\langle R_{t} e_{3}, q\right\rangle,
$$

where $(q, p) \in T^{*} S^{2}=P,\|p\|^{2}$ is the squared length of the covector $p$ with respect to the standard metric on $S^{2}$, and, $\langle$,$\rangle is the inner product on \mathbb{R}^{3}$. Also $g$ is the acceleration of gravity at the earth's surface, $e_{3}$ is the initial direction of gravity, and $m$ is the pendulum's mass. Roughly speaking, the $q$-coordinates are ones in which "directions are inertial," but positions are not. In these coordinates the direction of gravity, $R_{t} e_{3}$, is changing. The calculation of the Hamiltonian was done by starting with the Lagrangian (time-dependent in the $q$-coordinates) and performing the Legendre transformation. Also we used the usual linear approximation of the gravitational potential, and dropped a term quadratic in $\Omega$. For details of this calculation, see the forthcoming paper with Marsden and Ratiu (1988).

This Hamiltonian is the restriction of the family of completely integrable systems

$$
H: T^{*} S^{2} \times S^{2} \rightarrow \mathbb{R} ; \quad H(q, p ; x)=\frac{1}{2}\|p\|^{2}+\langle x, q\rangle
$$

to $T^{*} S^{2} \times \gamma, \gamma$ the circle of lattitude $\alpha$. The parameter space $S^{2}$ represents possible directions of gravity. For each frozen $x$, the group of rotations about $x$ leave $H$ invariant. Consequently, the angular momentum about the $x$-axis

$$
I(q, p, x)=\langle J(q, p), x\rangle
$$

is conserved, for $x$ frozen. Here $J(q, p)=q \times p$ is the moment map for the standard $S O(3)$ action on $T^{*} S^{2}$. I defines a family of Hamiltonian $S^{1}$ actions as in Example 1a, immediately above. [SO(3) acts on the parameter space $S^{2}$ in the standard way.] As already noted, the holonomy (4.1) of the associated HannayBerry connection gives (4.2). Note that in the course of one day the lab frame undergoes a rotation of $2 \pi$ relative to the $q$ frame. Thus to calculate the precession in the lab frame we should subtract $2 \pi$ from the $q$-precession, thus arriving exactly at Foucault's result (4.2).

The spherical pendulum is completely integrable, so instead of calculating the holonomy of the Hannay-Berry connection associated to the family of Hamiltonian $S^{1}$ actions, we could have done the calculation for the connection associated to a family of integrable systems. The result would be the same. In fact, the calculation would be the same, since the diagonal action of $S O(3)$ also leaves $H$ invariant (see Sect.5). Duistermaat (1980) has shown that the second action variable for the spherical pendulum cannot be globally defined, so this alternative calculation will not fit into the framework of this section (see Example 2, above). We would need the results (Theorem $1 \mathrm{~A}$ ) of the next section to make this integrable systems holonomy calculation rigorous. 


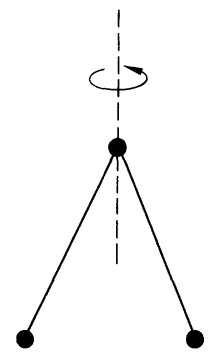

Fig. 2. An "azimuthal Foucault pendulum" spins quickly the axis of gravity. Its precession (relative to the prediction assuming constant azimuthal frequency) will be the same as the standard Foucault pendulum

Averaging is Unjustified for Foucault. $I=\dot{\theta}=0$ are the initial conditions for the standard Foucault pendulum. Therefore one cannot justify averaging over $\theta$, the azimuthal angle generated by the $S^{1}$ action. Averaging over the latitudinal angle $\varphi$ which measures the vertical swing of the pendulum is justified, as this oscillation is fast relative to the earth's rotation. Denote this averaging by $\langle\cdot\rangle_{\varphi}$. One finds

$$
\begin{aligned}
\langle d I / d t\rangle_{\varphi}=0 & \text { for oscillation (inside separatrix), } \\
\neq 0 & \text { for libration (outside separatrix, } \\
& \text { pendulum swinging all the way around) } .
\end{aligned}
$$

This makes it clear that the Hannay-Berry connection is not relevant for a highenergy Foucault pendulum. The nonzero average for libration can be made plausible by thinking of such a pendulum as a "Foucault gyroscope": total angular momentum $\mathbf{L}$ is then (approximately) conserved relative to an inertial frame, and hence its component $I$ in the lab frame will not be conserved.

Averaging about $\theta$ is justified if the pendulum has fast azimuthal rotation, i.e. I is large. This is very different from the standard pendulum, but could be constructed (see Fig. 2).

Example $1 b$. Essentially the same reasoning as in Example 1a leads to the result

$$
K \cdot \xi_{M}(x)=\left\langle J, \mathbb{P}_{x} \xi\right\rangle
$$

for the Hannay-Berry connection. Here $\mathbb{P}_{x}: \mathfrak{g} \rightarrow T_{x} M=\mathfrak{g}_{x^{\perp}}^{1}$ is orthogonal projection with respect to an Ad-invariant metric on $\mathfrak{g}$. We have also used this inner product to identify $g$ with $\mathrm{g}^{*}$, and hence the co-adjoint orbit $M$ with an adjoint orbit in $\mathfrak{g}$. The curvature form takes values in the Lie algebra of the maximal torus. The connection is the pull-back of the standard $G$-invariant connection on $G^{1} \rightarrow G^{1} / G$.

\section{Families of Completely Integrable Systems}

Most completely integrable system do not admit global action variables. For one, there are no action variables at points where some of the frequencies become infinite, for example, in a neighborhood of the separatrix for the planar 
pendulum, or more generally in a neighborhood of points on the stable or unstable manifold of a saddle point for the Hamiltonian. Even after deleting such points, most integrable systems do not admit global action variables. For example, the spherical pendulum does not (see Duistermaat, 1980). The obstruction to the existence of global action variables is called monodromy.

Families of completely integrable systems can exhibit a further problem. At certain parameter values there may be no local action variables, or even functions in involution, which are continuous in the parameter. An example is provided in Sect. 6 by a family of linear oscillators at a resonant parameter value.

As a consequence of these two difficulties, a general family of completely integrable systems does not admit a compatible family of Hamiltonian toral actions. Since we have no torus action to average over our previous definition of the Hannay-Berry connection fails. We will get around these difficulties by

$\left(1^{\text {st }}\right)$ deleting the set of points where the difficulties occur,

$\left(2^{\text {nd }}\right)$ thus obtaining a family of local Hamiltonian torus actions defined on the remaining good points,

$\left(3^{\text {rd }}\right)$ averaging over these local toral actions to construct the Hannay-Berry connection as before. action.

We will begin by being more precise about what we mean by a local torus

\subsection{Local Actions}

Definition. Let $Y$ be a manifold and $G$ a Lie group. By a local action of $G$ on $Y$ we mean an open cover $\left\{U_{\alpha}\right\}$ of $Y$ together with a collection of actions $\Phi_{\alpha}: G \times U_{\alpha} \rightarrow U_{\alpha}$ on each $U_{\alpha}$. Let $\operatorname{Aut}(G)$ denote the group of group automorphisms of $G$. We require that on the overlaps $U_{\alpha} \cap U_{\beta}$ there be smooth functions $\Phi_{\alpha \beta}: U_{\alpha} \cap U_{\beta} \rightarrow$ Aut $G$ such that

$$
\Phi_{\beta}(g, p)=\Phi_{\alpha}\left(\Phi_{\alpha \beta}(p) \cdot g, p\right) .
$$

The point is that the automorphisms $\Phi_{\alpha \beta}(p)$ of $G$ may be outer, and it may be impossible to piece the local actions on the $U_{\alpha}$ together in such a way as to form a global of $G$. But the average with respect to a local action is still well-defined, since Haar measure is invariant under outer automorphisms.

In the case of a completely integrable system the local torus action are defined by simply rotating the angles in a set of local action-angle variables. More precisely, let $f: P \rightarrow \mathbb{R}^{n}$ be the set of functions in involution, $\Sigma$ the set of $f$ 's critical values, and $C$ its image. Assume that the level sets of $f$ are compact. Using the flows of the components of the $f_{i}$ we obtain a local torus action on $P \backslash f^{-1}(\Sigma)$ in the above sense. The orbits of this local action are the connected components of the level sets of $f$. The cover $\left\{U_{\alpha}\right\}$ can be taken to be $\left\{f^{-1}\left(W_{\alpha}\right)\right\}$, where the $W_{\alpha}$ are simply connected and cover $C / \Sigma$. For further details see Duistermaat (1980).

Remark. There is an alternative bundle theoretic description of a local action. The $\Phi_{\alpha \beta}$ satisfy the cocycle condition for a bundle (provided the local actions are effective). In the completely integrable case the $\Phi_{\alpha \beta}$ are functions of the $f$ alone, consequently they define a torus-bundle over $C / \Sigma$. Its structure group is 
Aut $G=S l(n, \mathbb{Z})$. The bundle is equal to $T^{*}(C \backslash \Sigma) / \Gamma \rightarrow C \backslash \Sigma$, where $\Gamma \subset T^{*}(C \backslash \Sigma)$ is a bundle of lattices over $C / \Sigma$. The bundle "acts" fiber-wise on the bundle $P \backslash f^{-1}(\Sigma) \rightarrow C \backslash \Sigma$.

\subsection{Deleting Points}

Definition. A point $\left(p_{0}, x_{0}\right) \in P \times M$ is good for a family of completely integrable systems if

(a) there exists a neighborhood $U \subset M$ of $x_{0}$, and a smooth family $f_{U}: P \times U \rightarrow \mathbb{R}^{n}$ of functions in involution for $H$,

(b) $f_{U}(\cdot, x)$ is proper in a neighborhood of $p_{0}$ for each $x$ in $U$,

(c) $p_{0}$ is a regular value of $f_{U}\left(\cdot, x_{0}\right)$.

The set of good points will be denoted $(P \times M)^{g d}$, and the set of parameters satisfying condition (a) by $M^{g d}$. Both are open sets. The projection of $(P \times M)^{g d}$ onto $M$ is $M^{g d}$.

It follows from the Arnol'd-Liousville theorem that the component of the level set of $f_{U}(\cdot, x)$ near $p_{0}$ is an $n$-torus. Let $\left\{\gamma_{1}\left(f_{U}(z, x), x\right), \ldots, \gamma_{n}\left(f_{U}(z, x), x\right)\right\}$ denote an $n$-tuple of loops in $P \times\{x\}$ which represent a basis for the homology of the torus through $(p, x)$ and which varies smoothly as both the constant $f_{U}(p, x)$ and $x$ vary. Let $\beta$ denote a parameter-independent one-form on $P$ satisfying $d \beta=\omega$ near $\left(p_{0}, x_{0}\right)$. (In case $P$ is a cotangent bundle, $\beta=\Sigma p_{i} d q^{i}$ is the standard choice.) Then

$$
I_{j}(p, x)=\int_{\gamma_{J}\left(f_{U}(p, x), x\right)} \beta
$$

defines the standard action variables. They are smooth in both $p$ and $x$. The angle variables $\theta$ are constructed, as usual, by integrating the flows of the $I$, and so are also smooth in both variables. This local flow defines a toral action near $\left(p_{0}, x_{0}\right)$. In our parameter-dependent coordinates this action reads $(\theta, I, x) \mapsto(\theta+\varphi, I, x)$. This torus action depends on $f_{U}$, but only through the foliation of $(P \times U)^{g d}$ whose leaves are (components of) level sets of $f_{U}$ intersected with the fibers $(P \times\{x\})^{g d}$.

We now have a collection of torus actions on $(P \times M)^{g d}$, but not yet a local torus action. The problem is that there could be more than one choice of $f_{U}$ for a given parameter value $x_{0}$. This problem occurs in a family of linear oscillators at a resonance (Sect. 6). It is the reason that there are no smooth parameter dependent functions in involution near a resonant parameter value. To get around this final difficulty we make

Assumption 1. On the set of good points there exist $n$ smooth global functions in involution $f=\left(f_{1}, f_{2}, \ldots, f_{n}\right):(P \times M)^{g d} \rightarrow \mathbb{R}^{n}$ compatible with the given family of completely integrable systems $H: P \rightarrow \mathbb{P}$.

With Assumption 1 in place, there is a uniquely defined local torus action in a neighborhood of every good point. Set

$$
\mathscr{F}=\text { foliation of }(P \times M)^{g d} \text { corresponding to this local torus action. }
$$

The leaves of $\mathscr{F}$ are the connected components of the intersections of level sets of $f$ with the fibers $(P \times\{x\})^{g d}$. Now, at last, we can average. If $\alpha$ is any tensor defined 
along a leaf of $\mathscr{F}$ its average, $\langle\alpha\rangle$, is a tensor which is invariant under the local torus action.

Remark. We do not actually need to find action-angle variables to calculate averages. They can also be obtained by averaging with respect to the flows of the $f_{i}$.

Now define the Hannay-Berry connection by formula (2.1). It is defined on $(P \times M)^{g d} \rightarrow M^{g d}$, which in general is only a submersion, and not a fiber bundle.

Before translating the results of the previous sections to the present context, we must first better understand the requirement $\left\langle d_{M} I\right\rangle=0$. It is well-known that the standard actions satisfy this requirement. [A proof of this can be pieced together from Arnold (1978). For an alternative proof suggested by the paper (1988) of Alan Weinstein, write the author.] The following is easily verified.

Lemma. (1) $A$ set of ( $f$-compatible) action variables satisfies $\left\langle d_{M} I\right\rangle=0$ if and only if it is a standard action. Any two such actions are related by $I^{\prime}=m \cdot I+c$, where $m \in \operatorname{Sl}(n, \mathbb{Z})$ and $c \in \mathbb{R}^{n}$ are constant.

(2) The foliation $\mathscr{F}_{I} \supset \mathscr{F}$, whose leaves are locally the level sets of standard actions, is a globally well-defined foliation on $(P \times M)^{g d}$.

Remark 4.3. $\mathscr{F}_{I}$ is the pull-back of Weinstein's (1988) "isodrastic foliation" on the space of all Lagrangian tori embedded in $P$.

Result. To translate any result from Sects. 2 or 3 to the present context simply replace $P \times M$ with $(P \times M)^{g d}$ and the family of moment maps $I$ by the foliation $\mathscr{F}_{I}$, (equivalently, the collection of local standard actions $I$ ).

The definition of a Hannay-Berry connection and Theorem 2 needs no comment. The results of Sect. 3.5 on symmetry are valid even if we assume invariance of the functions $f$ (instead of $I$ ) under the diagonal action of $G^{1}$. We must be a bit more careful with Theorem 1. Let

$$
E^{I}=\text { a leaf of } \mathscr{F}_{I} .
$$

It is possible that the local torus action has "monodromy" in the parameter direction, as well as in the phase-space direction. (We do not know of any such examples which also satisfy our Assumption 1.) Consequently, it may be that the local torus action on $E^{I}$ cannot be made global, so that $E^{I}$ is still not a principal bundle. We will assume this difficulty away.

Theorem 1A. Suppose that $H: P \times M \rightarrow \mathbb{R}$ is a family of completely integrable systems, and that Assumption 1 holds on $(P \times M)^{g d}$. Then the Hannay-Berry connection on $(P \times M)^{g d}$ induces a connection on $E^{I} \rightarrow M$. In local standard actionangle coordinates the holonomy of the connection is given by the Hannay angles. All of the formulas, and in particular the curvature formula, of Theorem 2 are valid.

Suppose moreover that $M$ is simply connected. Then

(1) $E^{I} \rightarrow M$ is a principal torus bundle,

(2) the Hannay-Berry connection restricted to $E^{I}$ is a connection in the principal bundle sense.

Proof. The assumption of simple connectivity guarantees that the local action has no monodromy in the $M$-direction, and that $E^{I} \rightarrow M$ has connected fiber. We can 
thus piece the local toral action on $E^{I}$ together to form a global fiber-transitive action on $E^{I}$. $E^{I}$ is thus a principal bundle. The proof now follows the lines of the proof of Theorem 1.

Remarks. 1. Julian Wess asked the author "Is the curvature of this connection independent of the normalization axiom (3)?". The answer is yes: any Ehresmann connection satisfying (1) and (2) has curvature $\Omega\left(v_{1}, v_{2}\right)=\left\langle\left\{K \cdot v_{1}, K \cdot v_{2}\right\}\right\rangle$. This can be seen by direct calculation:

$$
\left\langle\left\{K \cdot v_{1}, K \cdot v_{2}\right\}\right\rangle=\left\langle\left\{K \cdot v_{1}-\left\langle K \cdot v_{1}\right\rangle, K \cdot v_{2}-\left\langle K \cdot v_{2}\right\rangle\right\}\right\rangle .
$$

More generally the curvature is normalization-independent of the $G$-action makes $P$ into a non-abelian completely integrable system, or, in Guillemin and Sternberg's (1984) terminology, a "multiplicity-free space."

2. In case $M$ is not simply connected, the conclusions of Theorem $1 \mathrm{~A}$ may sometimes still be shown to hold, either by direct calculation of the monodromy in the parameter direction, or by other means, for example, by using additional symmetries, as in the next section.

\section{Families of Linear Oscillators}

Family of Two-Dimensional Oscillators (Fig. 3). The parameter space $M$ is the space of two-by-two positive definite symmetric matrices.

$$
H: \mathbb{R}^{2} \times \mathbb{R}^{2} \times M \rightarrow \mathbb{R}, \quad \text { given by } H(q, p, S)=\frac{1}{2}\left(\|p\|^{2}+q^{t} S q\right)
$$

defines a family of integrable systems on $P=\mathbb{R}^{2} \times \mathbb{R}^{2}$ with its standard symplectic structure. If $S$ has a double eigenvalue then there are no choices of the action variables which are continuous in a neighborhood of $S$. Let $\Sigma \subset M$ be the ray of matrices with double eigenvalues:

$$
\Sigma=\{\lambda \text { Id }: \lambda>0\} \subset M .
$$

The space of good parameters is $M^{g d}=M / \Sigma$, which is homotopic to $S^{1}$. The curvature of the Hannay-Berry connection is zero, but the holonomy around any loop encircling $\Sigma$ once is -1 . Berry obtained this result in the quantum case in his original (1984) paper. As is usual for quadratic Hamiltonians, the classical results carries over exactly to the quantum case.

From a physical point of fiew this holonomy is obvious. Imagine two identical planar oscillators oscillating in phase on two separate identically oriented platforms. Keep the first platform fixed while rotating the other platform by 180 degrees. This rotation represents the fundamental loop $\gamma$ about which we will parallel translate. The matrix parameter $S$ (eigenvalues and directions), for the second oscillator will be identical to those of the first after the rotation, but the second oscillator will be oscillating exactly out of phase from the first.

For $S \in M / \Sigma$, the actions are given by

$$
\begin{aligned}
& I_{1}(q, p, S)=\frac{1}{2}\left\{\left(\lambda_{1}(S)\right)^{-1 / 2}\left\langle p, e_{1}(S)\right\rangle^{2}+\left(\lambda_{1}(S)\right)^{1 / 2}\left\langle q, e_{1}(S)\right\rangle^{2}\right\}, \\
& I_{2}(q, p, S)=\frac{1}{2}\left\{\left(\lambda_{2}(S)\right)^{-1 / 2}\left\langle p, e_{2}(S)\right\rangle^{2}+\left(\lambda_{2}(S)\right)^{1 / 2}\left\langle q, e_{2}(S)\right\rangle^{2}\right\},
\end{aligned}
$$




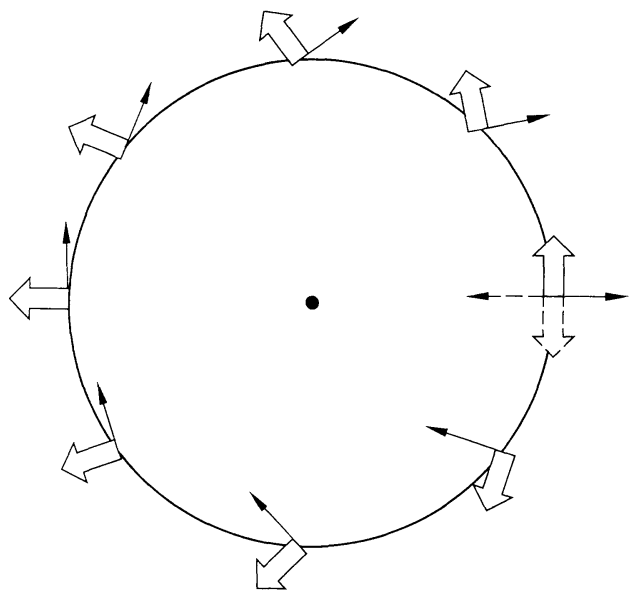

Fig. 3. Eigenvectors for $S=\left(\begin{array}{cc}a+x & y \\ y & a-x\end{array}\right)$ plotted in the $(x, y)$-plane with $a>0$ fixed

where $\lambda_{1}(S)>\lambda_{2}(S)$ are $S$ 's eigenvalues, and $e_{1}(S), e_{2}(S)$ the corresponding normalized eigenvalues, well-defined up to sign. $\left(I_{1}, I_{2}\right)$ are global smooth standard action variables on $\mathbb{R}^{4} \times M \backslash \Sigma$. They do not extend to $\mathbb{R}^{4} \times \Sigma$. This can be seen by direct calculation, or, more pictorially, by considering the maps

$$
e_{1}, e_{2}: M / \Sigma \rightarrow \mathbb{R} \mathbb{P}^{1}=S^{1}, \quad \text { by } S \mapsto\left\{ \pm e_{1}(S)\right\} \text { and } S \mapsto\left\{ \pm e_{2}(S)\right\} \text {. }
$$

(The \pm signs cannot be chosen consistently.) See Fig. 3. The calculations resulting in Fig. 3 are presented nicely by Takens (1979). After contracting $M / \Sigma$ to a circle, these maps each have degree 1 , hence cannot be continuously extended to $\Sigma$.

$G l(2)$ acts by symmetries on our family of integrable systems. It acts on $M$ by conjugation, and on $\mathbb{R}^{2} \times \mathbb{R}^{2}$ by $(q, p) \rightarrow\left(g \cdot q,\left(g^{t}\right)^{-1} \cdot p\right)$, which is the cotangent lift of the standard action on $\mathbb{R}^{2}$. The fact that the diagonal action on $P \times M \backslash \Sigma$ leaves the actions $I$ invariant is easily seen by breaking $G l(2)$ up into rotations, and dilations along the eigendirections. The rotations rotate the eigenvectors $e_{i}$ simultaneously with $q, p$, and consequently leaving the actions invariant. The dilations rescale the eigencomponents of $q, p$, and the eigenvalue in such a way as to leave the actions fixed.

In order to use Eq. (3.5) to calculate the connection we have to be able to average. Fix $S \in M \backslash \Sigma$, and let $q^{1}=\left\langle q, e_{1}\right\rangle, q^{2}=\left\langle q, e_{2}\right\rangle$ be the coordinates on $\mathbb{R}^{2}$ corresponding to the orthonormal eigenbasis. In these coordinates the angles $\theta^{i}$ conjugate to the actions $I_{i}$ are defined by

$$
q^{i}=\sqrt{2 I_{i} / \omega_{i}} \cos \theta^{i}, \quad p_{i}=\sqrt{2 I_{i} \omega_{i}} \sin \theta^{i},
$$

where $\omega^{i}=\sqrt[4]{\lambda_{i}}$ are the frequencies. Rotation of the angle $\theta^{i}$ by $\theta$ is the identity in the $q^{j}-p_{j}$ planes, $j \neq i$, and in the $q^{i}-p_{i}$ plane it is given by the matrix

$$
\left(\begin{array}{cc}
\cos \theta & \omega_{i}^{-1} \sin \theta \\
-\omega_{i} \sin \theta & \cos \theta
\end{array}\right) .
$$


The moment map for the $G l(2)$ action has components $q^{i} p_{j}$. For example, the moment map for the $S O(2)$ action is $J=q^{1} p_{2}-q^{2} p_{1}$, and for dilations the moment maps are $q^{1} p_{1}$ and $q^{2} p_{2}$. Using (6.3) one calculates

$$
\left\langle q^{i} p_{j}\right\rangle=0 \text {. }
$$

It follows from (3.5) that the horizontal lift of a generator $\xi_{M}$ is simply $\xi_{P} \oplus \xi_{M}$. The algebra of functions of the form $q^{i} p_{j}$ is closed under Poisson bracket. Consequently $\left\langle\left\{q^{i} p_{j}, q^{k} p_{l}\right\}\right\rangle=0$, and so the curvature of the Hannay-Berry connection is zero.

A loop in $M / \Sigma$ encircling $\Sigma$ can be generated by the $S O(2)$ action. The horizontal lift of this loop is then generated by the Hamiltonian function $K \cdot v=J-\langle J\rangle=J$, which just generates rotation in $P$. Since the loop in $M \backslash \Sigma$ is completed by rotating half-way around, its horizontal lift consists of rotating each $\mathbb{R}^{2}$ factor by $180^{\circ}$ yielding the holonomy:

Family of $n$-Dimensional Harmonic Oscillators. $H$ is given by the same formula, (6.1), with $(q, p) \in \mathbb{R}^{n} \times \mathbb{R}^{n}$, and $S \in M$, the space of $n \times n$ positive-definite symmetric matrices. $\Sigma$ is the set of matrices with multiple eigenvalues. Essentially the same calculation yields essentially the same result: the Hannay-Berry connection is flat but has nontrivial holonomy. The holonomy around a loop has the form

$$
-1 \oplus-1 \oplus \ldots-1 \oplus 1 \oplus \ldots \oplus 1,
$$

where each 1 or -1 acts on a four dimensional symplectic subspace of $\mathbb{R}^{2 n}$. This decomposition of $\mathbb{R}^{2 n}$ is determined by the decomposition of $\mathbb{R}^{n}$ into the onedimensional eigenspaces of $S$. A -1 or 1 occurs depending on whether or not these eigenspaces "switch places" an odd or even number of times as $\gamma$ is traversed.

The calculation can be done by the same method, replacing the symmetry group $G l(2)$ by $G l(n)$, or, conceptually simpler, $S O(2)$ by $S O(n)$ and the two dilations by $n$ dilations, one for each eigendirection.

\section{Proofs}

Throughout we will work along a fixed fiber $P \times\{x\}$, and $v \in T_{x} M$. We will continue to abuse notation by letting $v$ denote the vector field $0 \oplus v$ along $P \times\{x\}$. We also let $\lambda \in \mathfrak{g}$ denote its infinitesimal generator $\lambda_{P \times M}$.

Proof of Theorem 2. We first show that the Hannay-Berry connection satisfies the three defining axioms for a Hamiltonian connection.

Proof of Property (3), assuming the validity of property (2). Then $0=\langle\langle\mathrm{v}\rangle-\mathrm{v}\rangle=\left\langle X_{K \cdot v}\right\rangle=X_{\langle K \cdot v\rangle}$, so that $\langle K \cdot v\rangle$ is constant. This constant can be arranged to be zero.

Proof of Property (2). Write $g \in G$ in the form $g=\exp \lambda, \lambda \in \mathfrak{g}$. Using the Lie bracket formula (1.1) for $|\lambda, v|$ we find that

$$
\begin{aligned}
g^{*} v & =\int_{0}^{1} \frac{d}{d s}\left(\exp s \lambda^{*} v\right) d s+v=\int_{0}^{1} \exp s \lambda^{*}[\lambda, v] d s+v \\
& =\int_{0}^{1} \exp s \lambda^{*} X_{-d I^{\lambda} \cdot v} d x+v=X_{f}+v
\end{aligned}
$$


where

$$
f=-\int_{0}^{1} \exp s \lambda_{d I^{\lambda} \cdot v}^{*} d s
$$

is a function on $P$. The last step, going from the integral to $X_{f}$, is valid because $\exp s \lambda$ is fiber-wise symplectic. Now write $F(g, p)=f(p)$. There is more than one way to write $g=\exp \lambda$, so $F$ is only defined modulo constants. This constant can be fixed in a consistent smooth manner, for example by requiring $f\left(p_{0}\right)=0$ for some fixed $p_{0}$, or requiring that the integral of $f$ over $P$ vanish. Thus we have $\langle v\rangle=X_{K \cdot v}+v$, where

$$
K \cdot v(p)=\left\{\int_{G} F(g, p) d g\right\} / \operatorname{vol}(G) .
$$

This proves property (2) and even gives a formula for $K \cdot v$.

In order to prove property (1) we will first prove the Covariant Differentiation Formula; $D\langle\alpha\rangle=\langle D \alpha\rangle=\langle d \alpha\rangle \circ h$ for functions, $\alpha=f$,

$$
\begin{aligned}
D\langle f\rangle \cdot v & =\langle v\rangle[\langle f\rangle]=\operatorname{vol}(G)^{-2}\left\{\int_{G} g^{*} v d g\right\}\left[\int_{G} g^{*} f d g^{\prime}\right] \\
& =\operatorname{vol}(G)^{-2}\left\{\int_{G \times G} g^{*} v\left[g^{*} f\right] d g d g^{\prime}\right\} \\
& =\operatorname{vol}(G)^{-1}\left\{\int_{G}\langle v\rangle\left[g^{*} f\right] d g^{\prime}\right\} \\
& =\langle d f \cdot\langle v\rangle\rangle \quad \text { (using that }\langle v\rangle \text { is } G \text {-invariant). }
\end{aligned}
$$

And $\langle d f \cdot\langle v\rangle\rangle=\langle D f \cdot v\rangle=\langle d f\rangle(h \cdot v)$.

The proof for $k$-forms can be done the same way, except with more integrals. Alternatively, it could be done by noting that all three operators are graded derivations with respect to the wedge product, and then checking the formula for one-forms "on $P$ " and "on $M$. .)

Proof of Property (1)

$$
\begin{aligned}
D I^{\lambda} \cdot v & =\left\langle d I^{\lambda} \cdot h \cdot v\right\rangle=\left\langle d I^{\lambda} \cdot\left(X_{K \cdot v}+v\right)\right\rangle \\
& =\left\langle d_{P} I^{\lambda} \cdot X_{K \cdot v}\right\rangle+\left\langle d_{M} I^{\lambda} \cdot v\right\rangle=\langle\lambda[K \cdot v]\rangle+\left\langle d_{M} I^{\lambda}\right\rangle \cdot v .
\end{aligned}
$$

As noted immediately following Eq. (3.3), $\langle\lambda[K \cdot v]\rangle=0$, being the average of the differential of a function in a group direction. Therefore $D I=0$ if and only if $\left\langle d_{M} I\right\rangle=0$.

Proof of Curvature Formula. We prove the second formula. The first formula is a direct consequence of the second.

Extend the tangent vectors $v$ and $w$ at $x$ to vector fields. Write $v=\partial_{1}, w=\partial_{2}$, and $K \cdot v=K_{1}, K \cdot w=K_{2}$. If $Y \in T(P \times X)$, then its vertical projection is $Y-h \cdot T \pi \cdot Y$. The curvature is defined by

$$
\Omega\left(\partial_{1}, \partial_{2}\right)=\operatorname{vert}\left(\left[h \cdot \partial_{1}, h \cdot \partial_{2}\right]\right) \in C_{G}^{\infty}(P \times\{x\}),
$$

where "vert" means vertical projection composed with the identification of the resulting $G$-invariant Hamiltonian vector field with its $G$-invariant Hamiltonian function. 
Now

where

$$
\begin{aligned}
{\left[h \cdot \partial_{1}, h \cdot \partial_{2}\right] } & =\left[X_{K_{1}}+\partial_{1}, X_{K_{2}}+\partial_{2}\right] \\
& =\left[X_{K_{1}}, X_{K_{2}}\right]+\left[\partial_{1}, X_{K_{2}}\right]=\left[\partial_{2}, X_{K_{1}}\right]+\left[\partial_{1}, \partial_{2}\right] \\
& =X_{F_{12}}+\left[\partial_{1}, \partial_{2}\right]
\end{aligned}
$$

$$
F_{12}=-\left\{K_{1}, K_{2}\right\}+\partial_{1} K_{2}-\partial_{2} K_{1} .
$$

(The minus sign in the first term is correct: $\left[X_{K_{1}}, X_{K_{2}}\right]=X_{-\left\{K_{1}, K_{2}\right\}}$; the fact $\left[\partial_{1}, X_{f}\right]=X_{\partial_{1} f}$ was shown immediately following Eq.(1.1).)

Recall from Sect. 3.3 the definition of "almost generating": the function $\tilde{K} \cdot u$ almost generates parallel translation in the direction $u$ provided $d I \cdot u+\{I, \widetilde{K} \cdot u\}=0$. Also recall in this case $h \cdot u=X_{\tilde{K} \cdot u}+u-X_{\langle\tilde{K} \cdot u\rangle}$. It follows that $\operatorname{vert}\left(X_{\tilde{K} \cdot u}+u\right)=\langle\tilde{K} \cdot u\rangle$. Now $F_{12}$ necessarily almost generates parallel translation in the direction $\left[\partial_{1}, \partial_{2}\right]$. Consequently,

$$
\left\langle K_{2}\right\rangle=0 \text {, so }
$$

$$
\Omega\left(\partial_{1}, \partial_{2}\right)=\left\langle F_{12}\right\rangle,
$$

$$
\begin{aligned}
0 & \left.=D_{1}\left\langle K_{2}\right\rangle=\left\langle D_{1} K_{2}\right\rangle \quad \text { (we showed above that } D\langle\rangle=\langle D\rangle\right) \\
& =\left\langle\left\{K_{2}, K_{1}\right\}+\partial_{1} K_{2}\right\rangle .
\end{aligned}
$$

Hence $\left\langle\partial_{1} K_{2}\right\rangle=\left\langle\left\{K_{1}, K_{2}\right\}\right\rangle$. Similarly, $-\left\langle\partial_{2} K_{1}\right\rangle=\left\langle\left\{K_{1}, K_{2}\right\}\right\rangle$. Thus $\left\langle F_{12}\right\rangle=$ $-\left\langle\left\{K_{1}, K_{2}\right\}\right\rangle+\left\langle\left\{K_{1}, K_{2}\right\}\right\rangle+\left\langle\left\{K_{1}, K_{2}\right\}\right\rangle=\left\langle\left\{K_{1}, K_{2}\right\}\right\rangle$.

Proof of Proposition 2. Let $\lambda \in \mathrm{g}$ be an element that generates an $S^{1}$ subgroup, $S^{1}(\lambda) \subset G$, so that $e=\exp \lambda$ is the identity element. Apply the integral formula for $g^{*} v$ found in the proof of Theorem 2 to the case $g=e=\exp \lambda$. We have $v=e^{*} v=X_{f}+v$. Consequently, $X_{f}=0$, so that $f=$ a constant function on $P$, where $f=-\oint_{S^{1}(\lambda)} d I^{\lambda} \cdot v$. It follows that $\left\langle d_{M} I^{\lambda}\right\rangle \cdot v=$ const, since by Fubini's theorem we can do the integral over $G$ by considering $G$ as the bundle $G \rightarrow G / S^{1}(\lambda)$ and doing the integral over the $S^{1}$ fibers first. This fiber integral is the line integral we just did, so all these fiber integrals are constants (perhaps depending on the fiber); consequently the integral over all of $G$ is a constant. Since $g$ has a basis consisting of elements which generate $S^{1}$ 's, we have shown that $\left\langle d_{M} I\right\rangle$ is independent of $P$, hence is the pull-back of a one-form on $M$.

In order to see that this one-form is closed, we will use the same notation we used above in calculating the curvature. Let $\left\{x^{a}\right\}$ be coordinates on $M$. Then the $d x^{1} \wedge d x^{2}$ component of $d\left\langle d_{M} I\right\rangle$ is $\partial_{1}\left\langle\partial_{2} I\right\rangle-\partial_{2}\left\langle\partial_{1} I\right\rangle$. Now we have just shown that $\left\langle\partial_{2} I\right\rangle$ is a function of $M$ alone. Therefore $\partial_{1}\left\langle\partial_{2} I\right\rangle=D_{1}\left\langle\partial_{2} I\right\rangle$. Also $\left\langle\partial_{2} I\right\rangle$ $=\left\langle D_{2} I\right\rangle$, since, as we have already shown [immediately following Eq. (3.3)], $\left\langle\left\{I, K_{2}\right\}\right\rangle=0$, and in fact $\langle\{I$, anything $\}\rangle=0$. Thus

$$
\begin{aligned}
\partial_{1}\left\langle\partial_{2} I\right\rangle-\partial_{2}\left\langle\partial_{1} I\right\rangle & =\left\langle D_{1} D_{2} I\right\rangle-\left\langle D_{2} D_{1} I\right\rangle=\left\langle\left(D_{1} D_{2}-D_{2} D_{1}\right) I\right\rangle \\
& =\left\langle\left\{I, \Omega\left(\partial_{1}, \partial_{2}\right)\right\}\right\rangle=0 .
\end{aligned}
$$

\section{Generalizations and Future Directions}

Generalization to Symplectic Fiber Bundles. Replace $\pi: P \times M \rightarrow M$ by a symplectic fiber bundle $\pi: S \rightarrow M$ and $\omega \oplus 0$ by a closed two-form $\omega$ on $S$ whose restriction to 
each fiber is symplectic. See Gotay et al. (1980) for definitions and properties of symplectic fiber bundles. There is a natural Ehresmann connection associated with $\omega$, namely

$$
\text { Hor }_{s}=\text { the } \omega \text {-orthogonal complement to } \operatorname{ker} T \pi_{s}, \text { for } s \in S
$$

defines a horizontal distribution. We will denote the associated horizontal lift by $h_{0}$. As shown in the last few pages of Gotay et al., parallel translation with respect to $h_{0}$ is a (perhaps only locally defined) symplectic map from fiber to fiber.

Suppose $G$ acts on $S$ by symplectic fiber bundle automorphisms which cover the identity. Thus $G$ preserves the restriction of $\omega$ to each fiber, but not necessarily $\omega$. Also suppose that this action admits a fiber-wise moment map $I: S \rightarrow \mathrm{g}^{*}$. Then $h \cdot v=\left\langle h_{0} \cdot v\right\rangle$ defines the Hannay-Berry connection on $S$. The defining axioms (1), (2), and (3) for a Hamiltonian connection all make sense, provided we replace $0 \oplus v$ by $h_{0} \cdot v$. All of our results, in particular Theorem 2, still hold in this generality.

Holonomy in the Nonintegrable Case. As mentioned in Sect. 2.3, the bundle of symplectic reduced spaces,

$$
I^{-1}(\mu) / G_{\mu} \rightarrow M
$$

will play a crucial role in the non-integrable case. The fibers of this bundle are symplectic manifolds, and the reduced dynamics on each fiber (induced, say, by a given $G$-invariant family of Hamiltonians) can be very non-trivial. In order to (approximately) reconstruct the original dynamics on $I^{-1}(\mu)$ from these reduced dynamics we will need a consistent way of lifting curves from the reduced spaces, i.e. we will need fiber-wise connections on $\left(I^{-1}(\mu) \cap P \times\{x\} \rightarrow\left(I^{-1}(\mu) / G_{\mu}\right)_{x}\right.$, for $x \in M$. In a future paper with Marsden and Ratiu we propose to use a construction of Kummer (1981) to construct these fiber-wise connections.

These fiber-wise connections, together with the Hannay-Berry connection on $I^{-1}(\mu) \rightarrow M$, induce a connection on $I^{-1}(\mu) \rightarrow I^{-1}(\mu) / G_{\mu}$. We feel that the holonomy of this pieced-together connection, together with some information regarding the reduced dynamics, will yield useful information regarding the original dynamics.

Slowly Moving Constrained Systems. Many of the interesting physical examples of the Hannay angles, for example the rotating hoop, fall into this category. Strictly speaking, this category does not fit the framework of a slowly varying family of Hamiltonians. (Hannay and Berry made the hoop fit by replacing its moving constraint by a moving strongly constraining potential on a larger configuration space.) In the previously announced paper with Marsden and Ratiu, we will show how the notion of a Cartan connection on the space of motions of the constrained submanifold leads to another type of holonomy, also relevant to adiabatic motion. In cases where the group $G$ of the present paper is an isometry group of the constraint manifold we will relate the Cartan and Hannay-Berry connections. would not have embarked on this project. In the Introduction I explained my debt to Alan Weinstein, and to Jerry Marsden and Tudor Ratiu, for some ideas. At a summer conference in Boulder, I had a very useful conversation with Richard Cushman. In hindsight, I see that he was advocating something along the lines of our formula (2.1). I would also like to thank the organizers 
of that conference, Don Saari and Ken Meyer; and to thank Eugene Lerman, Tom Mrowka, Steve Zelditch, and Victor Guillemin for helpful conversations. This work was supported in part by NSF grant DMS-87-02502, and in part by my wife Judith's patience while in the middle of a move.

\section{References}

Anandan, J.: Geometric angles in quantum and classical physics. Phys. Lett. A (in press 1988) Atiyah, M.F.: Convexity and commuting Hamiltonians. Bull Lond. Math. Soc. 14, 1-15 (1982) Arnold, V.I.: Mathematical methods of classical mechanics. Berlin, Heidelberg, New York: Springer 1978

Arnold, V.I.: Geometrical methods in the theory of ordinary differential equations. Berlin, Heidelberg, New York: Springer 1983

Berry, M.: Quantal phase factors accompanying adiabatic changes. Proc. R. Soc. Lond. A 392, 45 (1984)

Berry, M.: Classical adiabatic angles and quantal adiabatic phase. J. Phys. A 18, 15-27 (1985)

Duistermaat, J.J.: On global action-angle coordinates. Commun. Pure Appl. Math. 33, 687-706 (1980)

Gerbert, P.: A systematic derivative expansion of the adiabatic phase. M.I.T. preprint., Center for Theoretical Physics (1988)

Gotay, M., Lashof, R., Sniatycki, J., Weinstein, A.: Closed forms on symplectic fiber bundles. Commentarii Math. Helv. 58, 617-621 (1983)

Guillemin, V., Sternberg, S.: Symplectic techniques in physics. Cambridge: Cambridge University Press (1984)

Hannay, J.H.: Angle variable holonomy in adiabatic excursion of an integrable Hamiltonian. J. Phys. A 18, 221-230 (1985)

Jackiw, R.: Three elaborations on Berry's connection, curvature, and phase. Preprint (1987)

Koiller, J.: Some remarks concerning Berry's phase. Seminario Brasileiro Analise (conference proceedings) SBA 26 (1987a)

Koiller, J.: The Foucault pendulum: an example of Berry's classical adiabatic angles. Preprint (1987b)

Koiller, J.: Classical adiabatic angles for slowly moving mechanical systems. Preprint (1988)

Kummer, M.: On the construction of the reduced phase space of a Hamiltonian system with symmetry. Indiana U. Math. J. 30, 2, 281-291 (1981)

Marsden, J., Weinstein, A.: Reduction of symplectic manifolds with symmetry. Rep. Math. Phys. 5, $121-130(1974)$

Marsden, J., Montgomery, R., Ratiu, T.: Hannay's angles for non-integrable and constrained systems. In progress (1988)

Simon, B.: Holonomy, the quantum adiabatic theorem, and Berry's phase. Phys. Rev. Lett. 51,

$2167(1983)$

Takens, F.: Motion under the influence of a strong constraining potential. In: Global dynamical systems. Lecture Notes in Mathematics, vol. 819, p. 425 445. Berlin, Heidelberg, New York: Springer 1979

Vinet, Luc: Invariant Berry Connections. U. of Montréal preprint (1987)

Weinstein, A.: Connections of Berry and Hannay type for moving Lagrangian submanifolds.

Preprint (1988)

Communicated by B. Simon

Received April 14, 1988; in revised form July 12, 1988

Note added in proof. The proof in Sect. 7 of the validity of Property 1, $D I=0$, is incorrect for the non-abelian case. (This is because $\langle I\rangle \neq I$.) Property 1 still holds in this case, and can be proved as follows. Note that $[\langle v\rangle, \lambda]=0$, and apply this Lie bracket to an arbitrary function $f$ to conclude that $D_{v} I^{\lambda}$ is constant. The proof then continues as before. 
2nd Note added in proof. The author has just received a preprint of Golin, Knauf, and Marmi entitled "The Hannay Angles: Geometry, Adiabaticity, and an Example". Their main result, Theorem 2, is almost identical to ours. In addition they have obtained some estimates regarding the accuracy of the Hannay angle approximation. 